\title{
Persistent holes in a fluid
}

\author{
Florian S. Merkt, Robert D. Deegan, Daniel I. Goldman, Erin C. Rericha, and Harry L. Swinney \\ Center for Nonlinear Dynamics, The University of Texas at Austin, Austin, TX 78712
}

(Dated: September 4, 2018)

\begin{abstract}
We observe stable holes in a vertically oscillated $0.5 \mathrm{~cm}$ deep aqueous suspension of cornstarch for accelerations $a$ above $10 \mathrm{~g}$. Holes appear only if a finite perturbation is applied to the layer. Holes are circular and approximately $0.5 \mathrm{~cm}$ wide, and can persist for more than $10^{5}$ cycles. Above $a \simeq 17 \mathrm{~g}$ the rim of the hole becomes unstable producing finger-like protrusions or hole division. At higher acceleration, the hole delocalizes, growing to cover the entire surface with erratic undulations. We find similar behavior in an aqueous suspension of glass microspheres.
\end{abstract}

The free surface of a fluid at rest in a container is flat. Departures from flatness induce a restoring flow whether the fluid is Newtonian, viscoelastic, or liquid crystalline: poke the surface, and the resulting indentation will be filled by the ensuing flow. In contrast, we have discovered that a vibrated aqueous suspension of cornstarch or glass microspheres can permanently support holes and vertical finger-like protrusions.

The catalog of interface morphologies in accelerated fluids is broad and well documented. Sinusoidal acceleration produces Faraday waves 1], solitons [2, 3], and jets [4]. Impulsive acceleration produces the RichtmyerMeshkov instability 5, 6], and continuous acceleration the Rayleigh-Taylor 7] instability, which are characterized by spires and bubbles [8]. The holes and fingers in vibrated cornstarch are distinct from these phenomena because they display neither the oscillation about a flat state of Faraday waves and solitons, the finite lifetime of jets, nor the unbounded growth of the RichtmyerMeshkov or Rayleigh-Taylor instabilities.

Figure 1 shows holes in a vibrated aqueous suspension of cornstarch (a-b) and glass microspheres (d-e). Depending on the container acceleration and frequency, the initial fluid surface is either flat or corrugated by Faraday waves. Above a critical acceleration, a finite localized perturbation of the fluid surface will grow into a stable cylindrical void that extends from the top to the bottom of the fluid layer. The most noteworthy feature of holes is their permanence: they do not close despite the hydrostatic pressure of the surrounding fluid, persisting as long as our observation ( $>10^{5}$ cycles). At yet higher accelerations, the holes lose stability to a temporally and spatially erratic state (see Fig. 1(c) \& (f)).

Experiment. A layer of cornstarch or glass microspheres in liquid was vertically oscillated sinusoidally with a frequency $f$ from 50 to $180 \mathrm{~Hz}$ and a peak acceleration $a$ up to $27 \mathrm{~g}$. The fluid container was round with a $9.4 \mathrm{~cm}$ diameter, and made from an aluminium base plate and acrylic sidewall. The container was attached to the shaker through an insulating rod to avoid heat transfer from the shaker to the container, and was sealed with an acrylic top to reduce evaporation. The layer depth was $0.5 \mathrm{~cm}$ for the cornstarch mixture and $0.2 \mathrm{~cm}$ for the glass microspheres. The peak acceleration of the container was controlled to within $\pm 0.01 \mathrm{~g}$.

The patterns were recorded with a 30 frames per second digital camera and a 1000 frame per second video camera. Lighting was provided by a ring of LEDs strobed

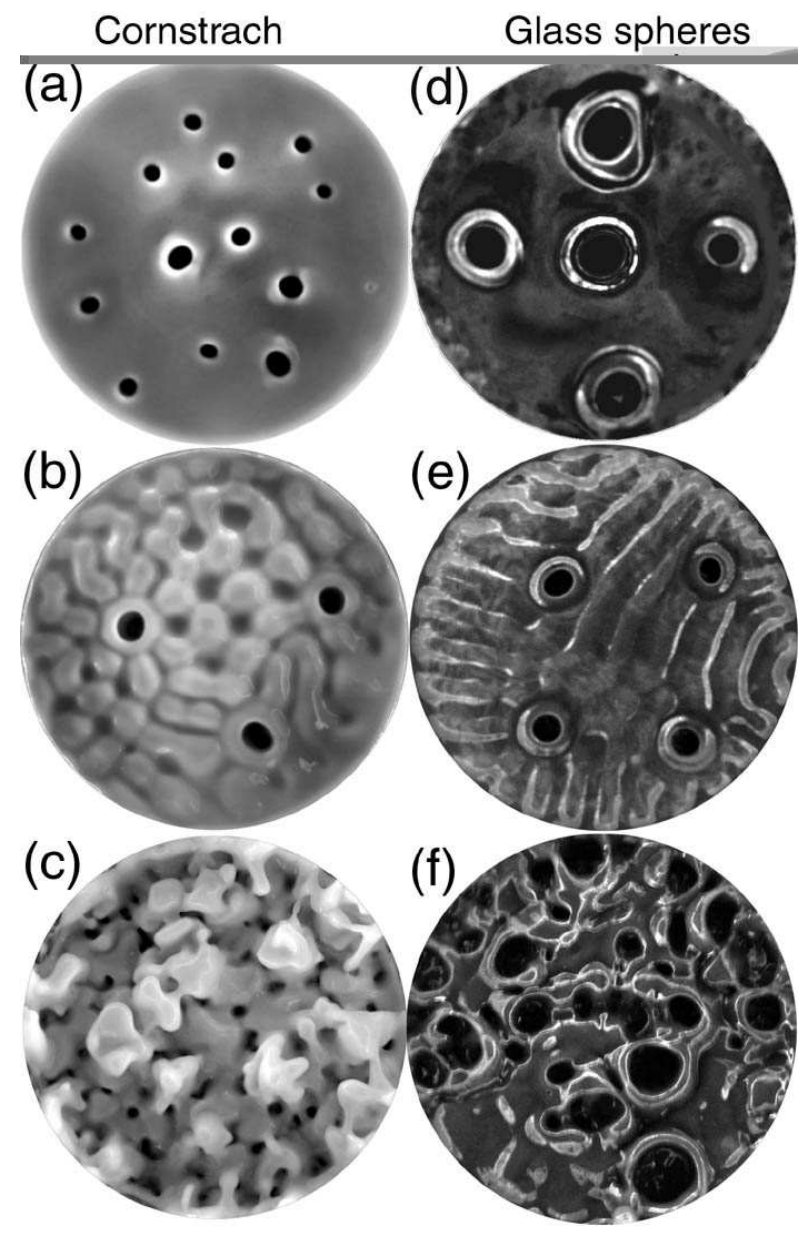

FIG. 1: Top view of a vibrated layer of (a-c) cornstarch and of (d-f) glass microspheres in liquids matched in density to the particles. Diameter is $9.4 \mathrm{~cm}$. White corresponds to the highest points, and black to depressions that reach the container bottom. Holes without Faraday waves: (a) $a=12 g$, $f=150 \mathrm{~Hz}$; (d) $30 \mathrm{~g}, 100 \mathrm{~Hz}$. Holes with Faraday waves: (b) $12 g, 60 \mathrm{~Hz}$; (e) $27.3 g, 92 \mathrm{~Hz}$. Delocalized state: (c) $30 g$, $120 \mathrm{~Hz}$; (f) $30 g, 60 \mathrm{~Hz}$. 
at $f$ or $f / 2$. The illumination was such that peaks on the surface appear bright and valleys dark.

We used unmodified, regular cornstarch consisting of $27 \%$ amylose and $73 \%$ amylopectin from Sigma Aldrich. The powder was dried at $50^{\circ} \mathrm{C}$ for a week and stored in a desiccator. A mixture was prepared daily by combining $23.22 \mathrm{~g}$ of cornstarch, and $36.78 \mathrm{~g}$ of a density-matched aqueous solution of $\mathrm{CsCl}$ with a density of $1.68 \mathrm{~g} / \mathrm{cm}^{3}$. The viscosity of the suspension was measured in a plateplate geometry with a Paar Physica TEK150 rheometer. Though all the quantitative results presented here are for cornstarch, we also used a glass microsphere and water mixture for the qualitative comparison in Fig. 1 The glass mixture was prepared with $29.37 \mathrm{~g}$ of $1-20 \mu \mathrm{m}$ glass balls from Jaygo (Union, NJ), and $18.36 \mathrm{~g}$ of an aqueous solution of sodium polytungstate with a density of $2.55 \mathrm{~g} / \mathrm{cm}^{3}$.

Hole dynamics and size selection. The interface of a vibrated fluid is flat at low accelerations and rippled by Faraday waves above a critical acceleration [1]. Holes form in the cornstarch mixture only if an indentation deeper than about $50 \%$ the layer depth and $0.4-2.0 \mathrm{~cm}$ wide is applied to the surface. We used a puff of compressed air delivered through a nozzle pointed at the surface to form a crater. The subsequent evolution of the crater depends on the parameters $a$ and $f$. For all parameter values explored, the crater may close within seconds. In the region of the phase diagram (Fig 2) marked "unstable" all craters are annihilated by this rapid decay. In regions marked "stable" and "metastable", the crater may develop into a hole: the crater adjusts to a preferred size (as shown in Fig 3) and remains fixed at that size for an extended period of time. At $f=120 \mathrm{~Hz}$ the characteristic diameter of the holes is $0.4 \mathrm{~cm}$ and at $f=180 \mathrm{~Hz}$ $0.6 \mathrm{~cm}$. Holes have a broad distribution of lifetimes, even for fixed $f$ and $a$; in the metastable phase the maximum lifetime is less than $10^{5}$ cycles and in the stable phase it is greater than $10^{5}$ cycles.

Holes collapse via one of two mechanism depending on their lifetime. Short lived holes collapse within a few seconds by a uniform contraction. Longer lived holes with lifetimes of order $10^{4}$ holes develop a hump on their rims, which then falls onto the hole either covering it or rendering it so small that it collapses rapidly by uniform contraction. On occasion this latter mechanism causes the hole to divide rather than collapse.

The cross-section of a hole is typically circular with a slight eccentricity. The shaft is smooth and slightly tapered towards the base. At the base of the hole, there is usually a ribbon of material about $0.1 \mathrm{~cm}$ high and wide that bisects the shaft. These features are not visible in Fig. 1 due to the lighting. At accelerations immediately above the stability line the material surrounding a hole remains level with the rest of the layer. At higher acceleration, the hole's rim rises about $0.05 \mathrm{~cm}$ above the surrounding fluid.

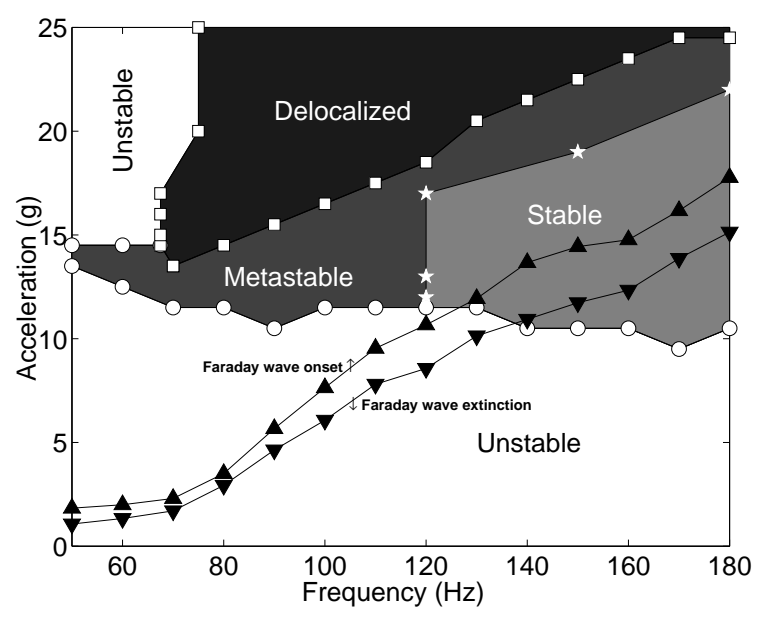

FIG. 2: Phase diagram for a vibrated aqueous cornstarch suspension as a function of acceleration and frequency. For accelerations above the $\circ$, a surface perturbation will form a hole. In the metastable regime holes always collapse within $10^{5}$ container oscillations; in the stable regime some holes endure for at least $10^{5}$ oscillations. show the acceleration threshold, below which holes collapse in less than $10^{4}$ cycles. In the delocalized regime the state is chaotic as shown in Fig. 1(c). Faraday waves appear with increasing acceleration at $\boldsymbol{\Lambda}$, and disappear with decreasing acceleration at $\boldsymbol{\nabla}$.

In addition to a slow meandering over hundreds of oscillations, hole size oscillates synchronously with the driving frequency, as shown in the inset of Fig. 3. The radius of a hole evolves as $r(t)=\bar{r}+\delta r \sin (2 \pi f t+\theta)$, where $\bar{r}$ is the mean radius, $\delta r$ is the amplitude of the oscillation, and $\theta$ is the phase relative to the container

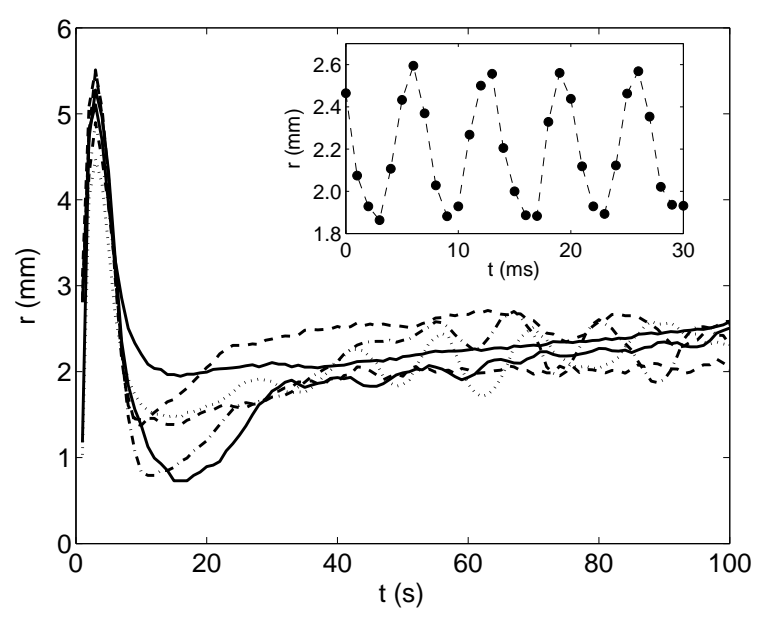

FIG. 3: Hole radius versus time in the cornstarch suspension for 6 different trials at $15 \mathrm{~g}$ and $150 \mathrm{~Hz}$. At $t=0$ a jet of gas was applied to the surface for $2 \mathrm{~s}$. The hole grows during this forcing, and immediately begins to shrink after the gas is cutoff. Inset: rapid oscillation of the hole. Note time scale. 
motion $z(t)=z_{o} \sin (2 \pi f t)$ where $z_{o}=-a /(2 \pi f)^{2}$ and $z$ is positive when the container is above its rest point. $\delta r$ is typically $0.15 \bar{r}$ and also varies by about $20 \%$ on a timescale of order $10^{3} f^{-1}$. The phase lag is shown as a function of frequency in Fig. 4.

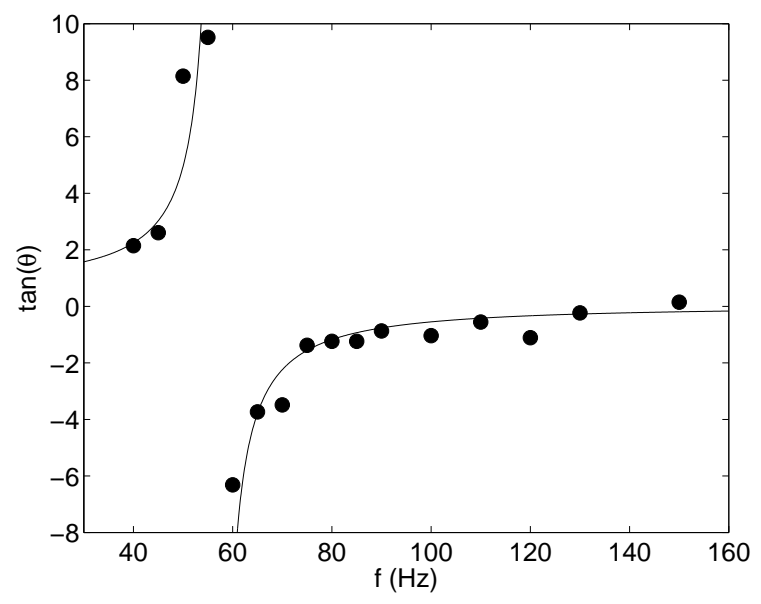

FIG. 4: The tangent of the phase lag versus frequency at $a=15 g$ The solid line is a fit to $\alpha /\left(1-\left(f / f_{o}\right)^{2}\right)$ where $\alpha$ and $f_{o}$ are fitting parameters.

Holes do not interact when their centers are separated by more than two diameters. Therefore, as shown in Figure 1(a), holes do not form regular patterns. Furthermore, they can be located anywhere in the container. Holes do occasionally come sufficiently close to interact, and do so by merging or one causing the other to collapse.

Faraday waves. Though Faraday waves are excited in cornstarch suspensions, the formation of holes is unrelated to these waves, as can be seen from the existence of holes when no Faraday waves are present, as in Fig.1(a) and (d). Furthermore, the phase boundaries for holes and Faraday waves are distinct. The Faraday transition is hysteretic and, moreover, the flat and surface wave state can coexist. As acceleration is increased at a fixed frequency, small patches of surface waves appear. The onset of these are indicated by the $\boldsymbol{\Lambda}$ in Fig. 2 As the acceleration is raised further, the patches grow and ultimately engulf the entire surface at values typical $50 \%$ higher than onset. Upon decreasing the acceleration, the surface waves breakup into patches which finally disappear at accelerations indicated by the $\boldsymbol{\nabla}$ in Fig. 2 Despite the ambiguity, there is a clear difference in the parameter dependence for the onset of holes and the onset of Faraday waves.

Delocalization. In the "delocalized" region in Fig. 2 a perturbation will generate a hole which immediately grows a protrusion from its rim. The sequence of photos in Fig. [5] shows the growth of such a structure. They are surprisingly tall and long-lived, reaching heights of $2 \mathrm{~cm}$ and remaining upright for thousands of oscillations. The

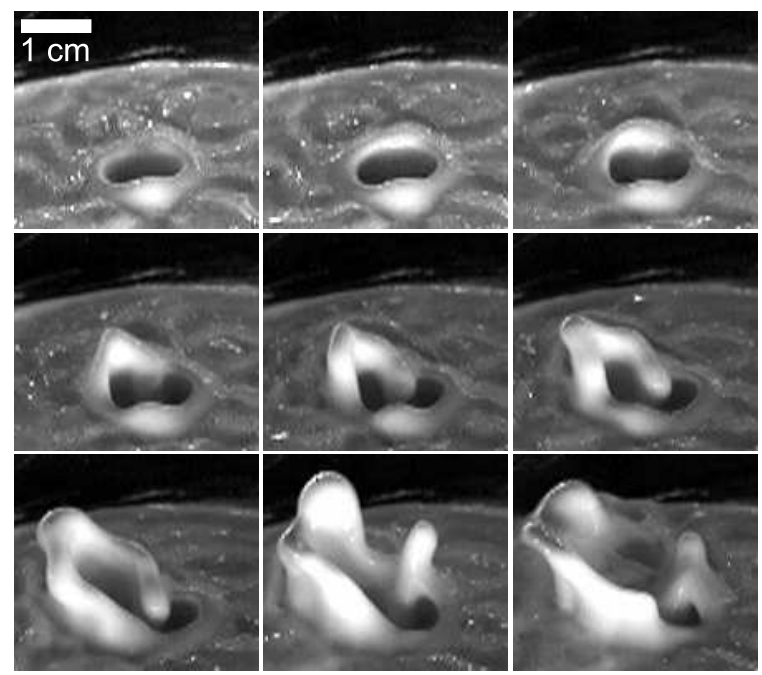

FIG. 5: Side view of the first steps of a delocalizing hole in cornstarch. These photographs were taken every $0.9 \mathrm{~s}$. Time increases from left to right and top to bottom. An initial hump on the rim begins growing upward, it reaches a maximum height, and then topples outward enlarging the area of fluid motion. This process is repeated until the entire surface of the liquid is active in the creation and destruction of vertical structures and voids (see Fig. 1(c)). These images were taken at $a=25 g$ and $f=80 \mathrm{~Hz}$.

protuberance eventually falls, nucleating a new hole, and the growth of a finger is repeated. After many repetitions the entire surface writhes with fingers and holes yielding the spatially and temporally erratic state shown in Fig. 1 (c) and (f).

Shear Thickening. We attribute the formation of holes to shear thickening, i.e., an increase of viscosity with shear rate. A plot of the viscosity for two identically prepared cornstarch suspensions is shown in Fig. 6 As is typical for shear thickening fluid [10], an initial decrease of the viscosity for increasing shear rate is followed by a rapid increase at a critical shear rate, which in this case is $\dot{\gamma}_{c}=8 \mathrm{~s}^{-1}$. Our conclusion is predicated on four observations: the critical shear rate is similar in magnitude to the shear rate at the throat of a hole; the phase lag of the holes radius relative to the driving force indicates the material response is primarily viscous; holes are unstable in a non-shear thickening fluid; and holes are stable in shear thickening fluids other than cornstarch suspensions.

The size of the hole varies synchronously with the driving frequency. The root-mean-squared shear rate at throat of the hole is approximately the interface speed, $(2 \pi / \sqrt{2}) \delta r f$, divided by a length scale of order the depth of the layer $h$. Using $\delta r / \bar{r} \approx 0.15, f=100 \mathrm{~Hz}$, and $\bar{r} / h \cong 0.4$ yields a shear rate $\dot{\gamma} \approx 27 \mathrm{~s}^{-1}$. The similarity of this value to the critical shear rate strongly suggests that shear thickening is an essential ingredient for holes.

The frequency dependence of the phase lag $\theta$ indi- 


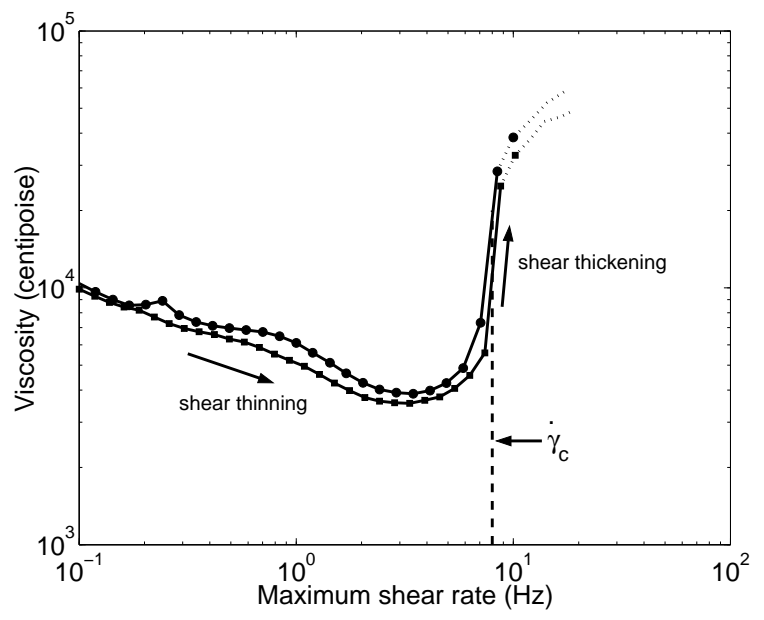

FIG. 6: Apparent viscosity versus maximum shear rate $\dot{\gamma}_{\max }$ for two identical samples of an aqueous cornstarch suspension. The torque $\tau$ on the plate was measured using a plate-plate geometry with a gap spacing $h$ of $0.10 \mathrm{~cm}$ and a plate radius $R$ of $2.50 \mathrm{~cm}$. The apparent viscosity was calculated as it would for a Newtonian fluid: $\eta=2 \tau /\left(\pi \dot{\gamma}_{\max } R^{3}\right)$. Since the shear rate increases with the radius, our measurement of the viscosity is a convolution of the geometry and the response of the fluid. The dotted line represents measured values that are not reliable due to an instability in the flow. The cornstarch samples were the same as in Fig. 1 and the temperature was kept at $27.6 \pm 0.2{ }^{\circ} \mathrm{C}$.

cates a combined viscous and elastic response of the fluid. Modelling the hole motion as a spring in parallel with a dashpot (i.e., a Voigt element, see 9$]$ for example) yields $\tan \theta=\alpha /\left(1-\left(f / f_{o}\right)^{2}\right)$, where $\alpha$ is a constant proportional to the dissipation and $f_{o}$ is the resonant frequency. As shown in Fig. 4 the data are well modelled by this equation; the resonance frequency is around $58 \mathrm{~Hz}$. Since the Voigt element is primarily viscous at high frequency, the phase data indicates that at frequencies above $60 \mathrm{~Hz}$ the material response becomes dominantly viscous; it is noteworthy that stable holes only form in this high frequency region.

We also tried to form holes in silicon oil (a Newtonian fluid) and various polybutadienes with viscosities 8.3, 28, and 72 Poise. In all cases, however, the initial crater was backfilled within a few hundred container oscillations.

In Barnes' review of shear thickening fluids, he asserts that any sufficiently concentrated suspension of solids in a fluid will shear thicken 10]. If our hypothesis that shear thickening is crucial for hole formation is correct, then any sufficiently dense suspension of solids ought to support persistent holes. For a dense suspension of glass microspheres this is indeed the case, as shown in Fig. 11(d) $\&(\mathrm{e})$.

Conclusions. We have shown that a vertically vibrated aqueous suspension of cornstarch displays a number of unexpected patterns-holes, fingers, and a delocalized state-that can only be attained by the application of a finite perturbation. Holes have a preferred size, vibrate synchronously with the container, and are unrelated to the well-known Faraday wave instability. At higher acceleration the hole becomes unstable to the creation of finger-like structures, which at even higher accelerations are responsible for the transition to a delocalized state in which the free surface is covered with fingers and holes. Our observation suggest that shear thickening is the key ingredient for hole formation. The outstanding questions are how do holes stay open, how can the finger like protrusions grow and remain unright, and what drives the transition to the delocalized state?

We thank M. Schröter, W.D. McCormick, P. Heil, A. Lee, W. Zhang for valuable suggestions, and P. Green and A. Jamieson for assistance with the viscosity measurements. We acknowledge support by the Engineering Research Program of the Office of Basic Energy Sciences of the U.S. Department of Energy under grant number DE-FG03-93ER14312. FSM acknowledges support by the Friedrich Ebert Stiftung.

[1] M. Faraday, Philos. trans. R. Soc. London 121, 319 (1831).

[2] O. Lioubashevski, H. Arbell, and J. Fineberg, Phys. Rev. Lett.76 3959 (1996).

[3] O. Lioubashevski, Y. Hamiel, A. Agnon, Z. Reches, and J. Fineberg, Phys. Rev. Lett. 83, 3190 (1999).

[4] M.S. Longuet-Higgins, J. Fluid Mech.127, 103 (1983).

[5] R.D. Richtmyer, Commun. Pure Appl. Math. 13, 297 (1960).

[6] E.E. Meshkov, Fluid Dyn. 4, 101 (1969).

[7] G.I. Taylor, Proc. Royal Society, A 201, 192 (1950).

[8] U. Alon, J. Hecht, D. Ofer, and D. Shvarts, Phys. Rev. Lett. 74, 534 (1995).

[9] J. D. Ferry, Viscoelastic Properties of Polymers (Wiley, 1961).

[10] H.A. Barnes, J. Rheol. 33, 329 (1989). 